\title{
Commutator-Operated Digital Caliper with Visual Display and Card-Punch Capacity
}

\author{
W. STUART HUNTER, * STANLEY M. GARN, and RICHARD L. MILLER \\ Center for Human Growth and Development, University of Michigan, Ann Arbor, Mich- \\ igan 48104
}

A variety of automatic and semiautomatic measuring devices with print-out, card-punch, and paper-tape capacity have been used in the analysis of lateral head radiographs, posteroanterior head radiographs, and oblique-jaw views. Such devices include the tape-printing GOAT, + the card punching GADRS-4 and GADRS-5, $\rightarrow$ and the OSCAR. $\$$ These automatic measuring instruments have satisfactory readout accuracy down to $0.1 \mathrm{~mm}$, are far faster than hand caliper measurements, and eliminate visual reading and transcription errors. However, the fixed $X$ and $Y$ coordinates render them relatively clumsy for measurements unrelated to a single reference line. Though dialreading calipers can be modified for electric digital display (S. M. GARN, R. H. HELMRICH, and A. B. LewIs, J Dent Res 46:306, 1967), full card-punching capacity is not obtained without additional circuitry and components.

As an alternative to the GADRS or OSCAR instruments, but using stock parts, use has been made of the Decitrak\$ (Illustration). Essentially, this instrument consists of two functional parts: (1) the measuring-encoding unit, and (2) the digitizing output unit. Together they interface with an IBM 526 Printing Summary Punch, eliminate visual read-out error and transcription mistakes, obviate the need for precoding forms, and yield punch cards with a time saving of $90 \%$ over hand methods.

The commutator-type encoding unit can be fitted with adjustable needle points for caliper micrometry, or with more conventional caliper

This investigation was supported in part by USPHS research grant DE00224 from the National Institute of Dental Research, National Institutes of Health, Bethesda, Md.

Additional informaticn available on request to authors.

Received for publication January 27, 1969.

* Present address: Faculty of Dentistry, University of Western Ontario, London, Ontario, Canada.

$\pitchfork$ Gerber Scientific Co., Hartford, Conn.

* Benson Lehner Corp., San Diego, Calif.

$\S$ Theta Scientific Co., Saddle Brook, New Jersey. jaws for measurements of tracings. $\|$ It can be placed at any angle over a radiograph or tracing and it can be set to give $\mathrm{X}$ or $\mathrm{Y}$ coordinate readings along the Frankfurt planes or at right angles to the planes. The entire unit is satisfactorily free from temporal or thermal drift. The digitizing unit is completely solidstate. The equipment exhibits minimal operational difficulties and interfaces with the IBM 526 Printing Summary Punch without bulky circuitry. Modifications have been made for the measurement of head radiographs of rhesus monkeys and for cortical thickness measurements of mandibles and small tubular bones, as well as for photographic and direct (optical) projections of histologic slides.

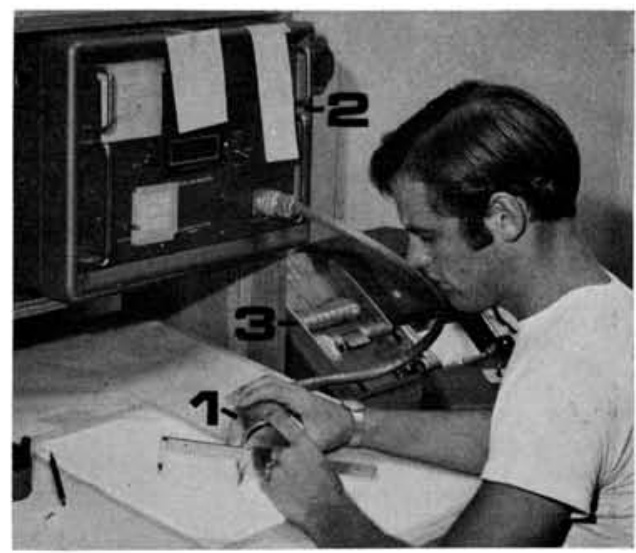

Decitrak unit in position for measurement on tracing showing (1) the hand-held encoding caliper, (2) the solid-state digitizing unit, and (3) the printing card-punch. A foot control activates the punch.

II The interface system was designed and built by Ian Scott and Kurt Coleman, and the caliper head fabrication by Sarns, Inc., Ann Arbor, Mich. 